\title{
A new remote monitoring device to track magnetic resonance imaging machine cooling system failures
}

\author{
Oussama Elallam, Mohamed Hamlich \\ Complex Cyber Physical Systems Laboratory, The National Superior School of Arts and Crafts, University Hassan II, Casablanca, \\ Morocco
}

\begin{tabular}{l} 
Article Info \\
\hline Article history: \\
Received Jun 2, 2021 \\
Revised Oct 29, 2021 \\
Accepted Nov 30, 2021 \\
\hline
\end{tabular}

Keywords:

Cooling system

Failure

IoT

MRI

Quench

\begin{abstract}
The magnetic resonance imaging (MRI) machine cooling system has a vital role in the conduct of MRI examinations because a shutdown of the MRI cooling system in the absence of the manipulators can lead to grave consequences over time, like quench, which is the vaporization of helium liquid in the MRI tank, and it's the most expensive MRI failure. To limit the risks of this problem, several companies have tried to develop a monitoring system to track MRI cooling system failures but all solutions proposed are complicated and demand many connections with MRI. The proposed solution is simple, easy, and efficient requires only one joint with the helium compressor, and it has a humidity and temperature sensor to detect quench incident, it works using an advanced monitoring algorithm that evaluates the status of the cooling system and identifies breakdowns, in case of failure our system will send short message service (SMS) notifications and emails to the customer service team. The proposed solution shows the potential for starting the research to understand the relationship between the behavior of the MRI cooling system and the quench using machine learning algorithms.
\end{abstract}

This is an open access article under the CC BY-SA license.

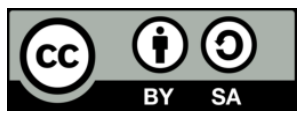

\section{Corresponding Author:}

Oussama Elallam

Complex Cyber Physical Systems Laboratory, The National Superior School of Arts and Crafts

University Hassan II

150 Boulevard du Nil, Casablanca 20670, Morocco

Email: elallamoussama7@gmail.com

\section{INTRODUCTION}

Magnetic resonance imaging (MRI) or magnetic resonance imaging is one of the most popular, reliable, and precise medical imaging techniques. It provides two- and three-dimensional views of inside the body and particularly useful for detecting diseases or disorders that conventional examinations have failed to identify. Typical MRI and nuclear magentic reasonance (NMR) machines produce diagnostic images by affecting the patient's gyromagnetic materials with controlled gradient magnetic fields and radiofrequency pulses in the presence of a main magnetic field developed by a superconducting magnet [1]-[5]. To ensure proper operation and accurate imaging, the magnet at the heart of an MRI machine must be kept at an ultralow temperature of 4 kelvin or $-269{ }^{\circ} \mathrm{C}$ [6]-[10]. To accomplish that liquid helium is used, which is cooled using a specialized compressor called a Cryo-chiller. There are many series of coils and heat exchangers that are used to cool the cryo-cooler, which is crucial because if the helium temperature increases, the machine may overheat, without cooling, within a few hours, all the helium will vaporize and escape through the safety valves, after which the magnet will suffer irreparable damage [11]-[15]. Helium refills cost tens of thousands of dollars, while the loss of the magnet can push the capital loss to several million.

The cooling of an MRI machine with a magnet has to stay at almost unbelievably low temperatures. It is an extreme example of cooling technology, which requires precise monitoring to keep a constant eye on 
the MRI cooling system to avoid losing helium liquid due to a failure in the machine. Also, this monitoring of the MRI cooling system will ensure that the MRI machine works at the desired level of performance.

Earlier conducted researches on MRI cooling system monitoring system have led to developing many inventions in this field, some of those inventions [16], [17] are based on analog/digital interface connected to many MRI machine parts, it compares the gathered data with previous alarm conditions and sends alert notifications in case of dysfunction. The invention in [18] proposes a remote monitoring system for mobile MRI machines of general electric healthcare, the proposed system connects to the magnet monitor to check if a failure has occurred, and if it's the case it will send a notification alert with the location of the mobile system. The invention in [19] presents a smart helium compressor with an integrated communication system that allows the compressor to communicate with the maintenance team using the local supervision system of the MRI machine or independently thereof.

Moreover, numerous commercial devices have been developed in the past to keep constant monitoring of the MRI cooling system and to provide a fast response in case of failure. One of the already existing devices "ColdTRAC" uses multiple connections with different parts of MRI to screen helium level, chiller temperature, cold head efficiency, magnet vessel pressure, compressor pressure, and chilled water flow. It can send alert notifications to 26 contacts in case of defeat of the cooling system. There is also a proposed system "Z-Pulse" that uses the same precedent approach but it can scan more parameters like MRI Magnet Shield temperatures, MRI field status, Equipment room temperature, and humidity. It provides continuous monitoring of the cooling system to detect any breakdown and notify the technical team by text messages and emails. The third proposed device "daVinci" does the same features as the two previous systems, except that it's equipped with a Global positioning system (GPS) localization module to be used in mobile MRI systems which will give the exact localization of the defeated MRI system to the technical team. Another existed device called Chiller Fault Notification System connects just to the Chiller as a simple solution to alert technical staff if a shutdown occurred in the chiller, it's plugged into the chiller errors port and observe the status of each pin which is related to a specific error, the system sends a short message service (SMS) notification in case of a failure.

The first three commercial devices are efficient, but they require many connections with the MRI system and special training to the maintenance team to use them in the best way, and they are costly. On the other side, the last system is simple, cheap and it demands no previous training to use it, but it is still insufficient since it covers only the chiller, which is just a part of the MRI cooling system. Here it's appaired the need for a simple, cheap, and efficient system that requires less connection with MRI machine system and covers all MRI cooling system failures.

The proposed solution in this research is a simple based Arduino remote monitoring system that requires only one connection with the MRI cooling system. It can detect all the cooling system failures and even the happening of the quench due to its Humidity and temperature sensor. Our proposed solution sends email and SMS notifications to alert the maintenance team if dysfunction has occurred. It is the only solution that combines effectiveness and simplicity and saving effort, energy, and money.

\section{RESEARCH METHOD}

The main challenge in this project was establishing a connection with the MRI cooling system simply and with no influence on the system's normal function. Therefore, we have studied every part of the MRI cooling system to understand how it works. Moreover, to count our possibilities to implement an external system for remote tracking.

A magnetic resonance imaging system -as shown in Figure 1 has a superconducting magnet housed in a cryostat. It has a cryogenic refrigerator that cools in the cryostat, a helium compressor that supplies compressed helium to the cryogenic refrigerator, and receives a compressed helium stream from the refrigerator. Also, it has a heat exchanger, or "cooler" (which pumps a mixture of water from the antifreezewater to cool the gradients and remove some heat from the helium system), and a magnet monitoring system controlling the operation of the resonance imaging system magnetic. Moreover, it has a device that controls the speed synchronization of the operation of the helium compressor according to predefined specifications of helium compressor rates in response to system status data [20].

After a deep analysis of the MRI cooling system, we have found that the helium compressor has an internal autodiagnosis system that checks if any failure has occurred. The helium compressor sends the results of its diagnosis to The MRI mainboard via a parallel communication with no specific protocol using a DB-25 cable plugged in an interface diagnostic of the compressor [21]. This diagnostic interface-as shown in Figure 2 is a DB-25 female port. Each pin of it is related to a specific error in the cooling system process. If an $\mathrm{x}$ failure has occurred, his pin will carry the logical level $24 \mathrm{~V}$, as shown in Table 1. 


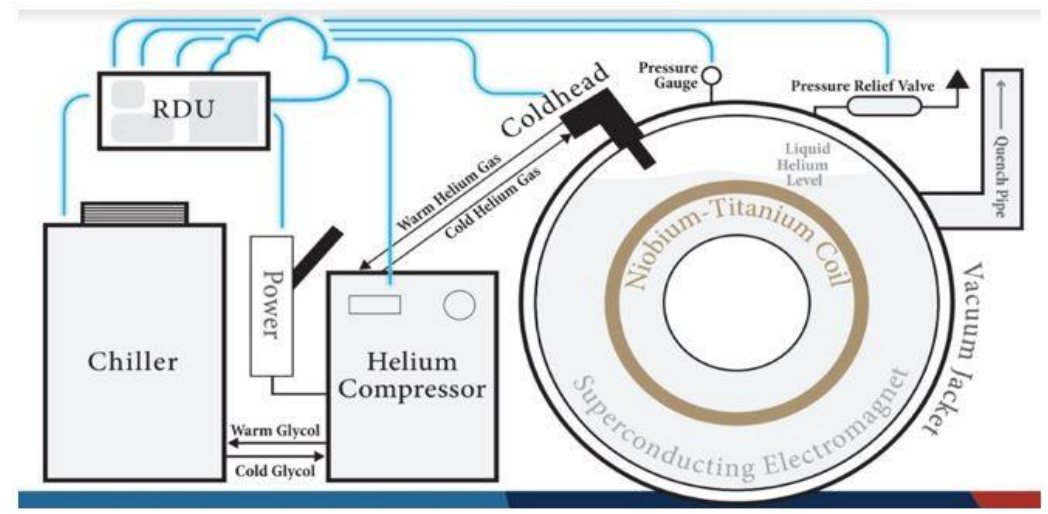

Figure 1. MRI cooling system

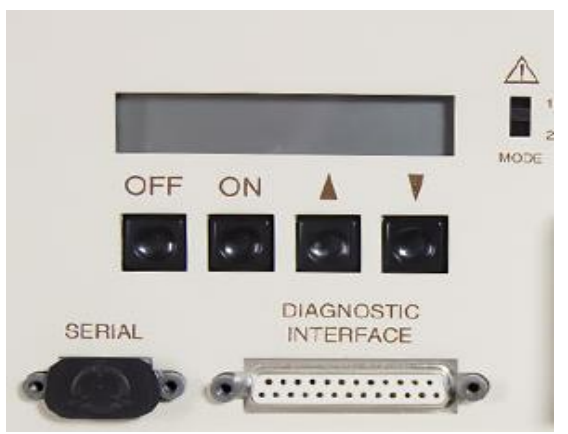

Figure 2. Diagnostic interface

Table 1. Diagnostic interface connector pin functions

\begin{tabular}{cl}
\hline Pin \# & \multicolumn{1}{c}{ Function } \\
\hline 14 & $\begin{array}{l}\text { High water temp. If the temperature of the water supply to the heat exchanger is too high, } \\
\text { this pin will carry a +24VDC, } 20 \mathrm{~mA} \text { max. signal. } \\
\text { Low water flow. If the temperature of the water leaving the heat exchanger is too high, this } \\
\text { pin will carry a +24VDC, 20 mA max. signal. }\end{array}$ \\
16 & $\begin{array}{l}\text { Low pressure shutdown. On a compressor return low pressure error }(<15 \text { psig) this pin will } \\
\text { carry a +24 VDC, 20 mA max. signal. }\end{array}$ \\
17 & $\begin{array}{l}\text { Power error. On a phase or fuse error, this pin will carry a +24VDC, } 20 \mathrm{~mA} \text { max. signal. } \\
\text { Gas temp error. On a high helium gas discharge temperature error, this pin will carry a } \\
\text { +24VDC, 20 mA max. signal. } \\
\text { Run status. When the compressor and cold head are running or the compressor is running } \\
\text { with the cold head paused, this pin will carry a +24VDC, 20 mA max. signal. }\end{array}$ \\
20 & $\begin{array}{l}\text { Motor winding temperature error. On a high compressor motor winding temperature error, } \\
\text { this pin will carry a +24VDC, 20 mA max. signal. }\end{array}$ \\
\hline
\end{tabular}

Therefore, the proposed system will connect to the helium compressor via a simple DB25 Y Splitter Parallel cable-DB25M to two DB25F as represented in Figure 3. The DB25 Y splitter is mandatory to ensure regular communication between the MRI machine and its cooling system. Our proposed system has a galvanic isolation circuit to ensure that no negative influence will occur on the normal function of the MRI cooling system.

The proposed system has a DB25 male port in input to connect to the helium compressor. Also, it has a humidity and temperature sensor to detect changes in the MRI room atmosphere, a $24 \mathrm{~V}$ to $5 \mathrm{~V}$ voltage adapter to detect the logic states of pins without burning the microcontroller, which is an Arduino mega that detects failures in the cooling system by analyzing input pins changes. In case of a failure in the cooling system, it sends SMS and emails due to a global system for mobile communications (GSM) module and a Wi-Fi module. Our system has an organic light-emitting diode (OLED) screen to display system status, a power management circuit, and a battery to keep working in case of losing power; All those components are presented in the block diagram in Figure 4. 


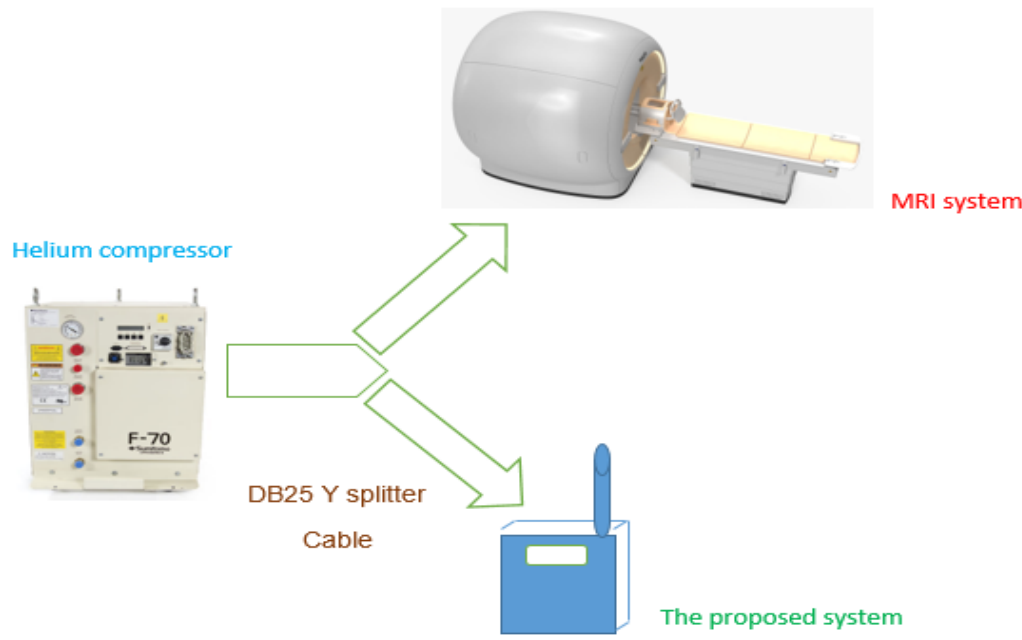

Figure 3. Connections diagram

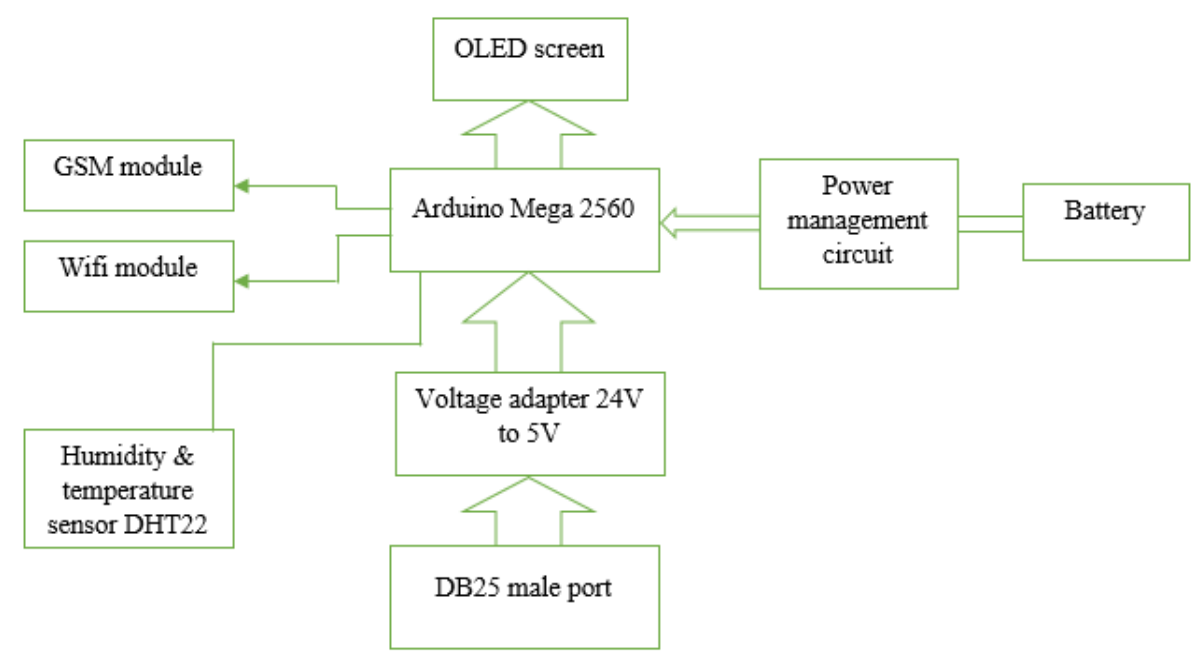

Figure 4. Block diagram of our system

\subsection{Hardware implementation}

Figure 5 shows the hardware architecture of the proposed system that consists of a voltage adapter $24 \mathrm{~V}$ to $5 \mathrm{~V}$ in the input; it's a group of parallel channels based on the octocopter isolation circuit connected to the digital inputs (\#23-\#29) of Arduino with pull-down configuration. The voltage adapter allows us to adapt the high logic level $24 \mathrm{~V}$ of the diagnostic interface with the high logic level $5 \mathrm{~V}$ of the Arduino to protect it from burning out. Moreover, the proposed system revolves around the Arduino Mega 2560 microcontroller board that analyzes the input pins status of the voltage adapter. Besides, the proposed system contains a humidity and temperature sensor connected to the Arduino in digital pin \#7 and placed above the MRI machine to monitor the air conditions and figure out if there is a sudden decrease in temperature and increase in humidity values, which means the quench has occurred.

The Arduino board does all the calculations to detect any failure in the MRI cooling system. If a malfunction has occurred, the Arduino will send SMS notifications using the sim8001 chip connected to the Arduino via serial communication in Tx1 and Rx1 pins of the Arduino and will send emails via a Wifi module which is, in our case, a Nodemcu Esp12e connected to the Arduino via a serial communication in Tx3 and Rx3 pins as illustrated in Figure 5. The proposed system has a small OLED screen driven by the Arduino board and connected to it in serial data (SDA) and serial clock (SCL) pins to print the error text to the maintenance team on-site next to the MRI cooling system to make the diagnostic operation easy. 


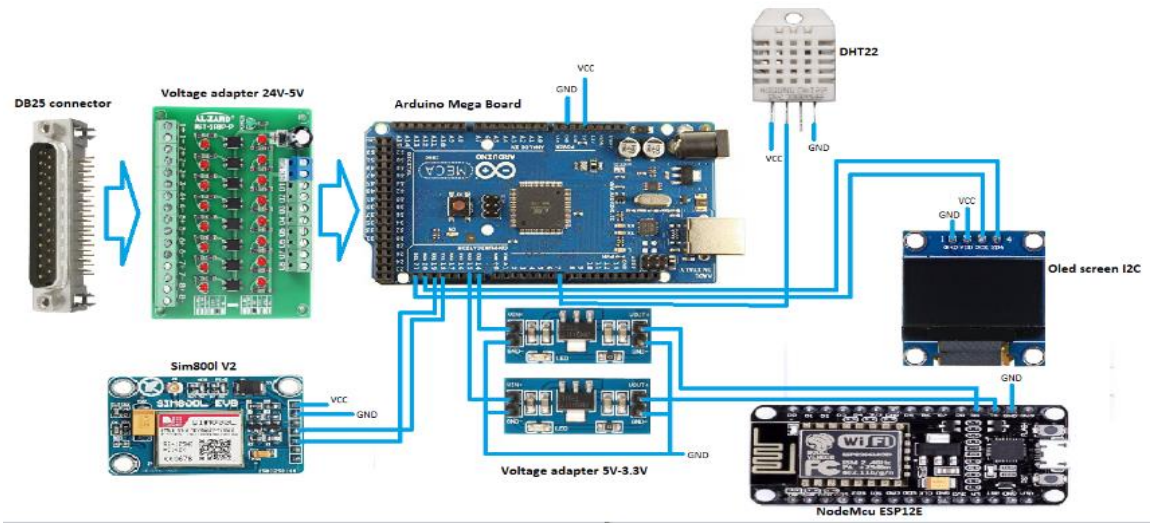

Figure 5. Hardware architecture

\subsection{Software implementation}

The software of our proposed system includes two parts, one for the Arduino Mega board to monitor the errors of the MRI cooling system and to send SMS notifications and also to communicate with the Nodemcu module, and the other part for the Nodemcu module to make it able to send emails in case of failure and to exchange information with the Arduino board. Figure 6 shows the flowchart of the Arduino code; first, the Arduino will measure the humidity and temperature values surrounding the MRI machine using a DHT22 sensor to detect any abnormal decrease and increase, which could be a significant sign of a quench incident. If all is good, the Arduino will start scanning the status of digital input pins to figure out if there's a state change to a high logic level. In this case, the Arduino will match the triggered pin with its failure code to print it in the OLED screen and send it to the Nodemcu for sending emails. Also, it will send an SMS notification with the specific failure code to the maintenance team. If it didn't receive a reception confirmation message in the next five minutes, it would send another SMS notification again and again until someone confirms the reception of its notification.

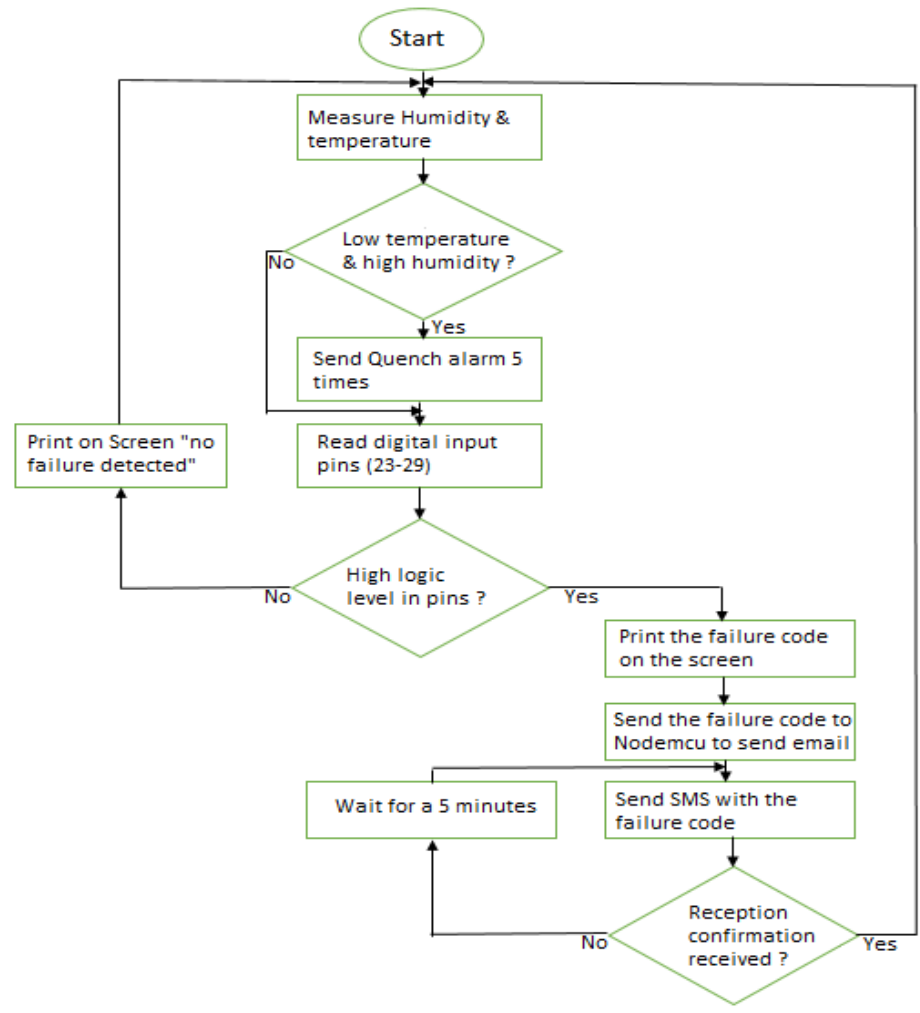

Figure 6. Flowchart of Arduino 
The Nodemcu board is in charge of enabling our system to connect to the internet. As shown in Figure 7, the Nodemcu will start by establishing a connection to a wifi network. After that, it will communicate with the Arduino board to know if any failure occurred; if it's so, the Nodemcu will match the failure code received from Arduino with a predefined email text dedicated to this failure and send it to the maintenance team.

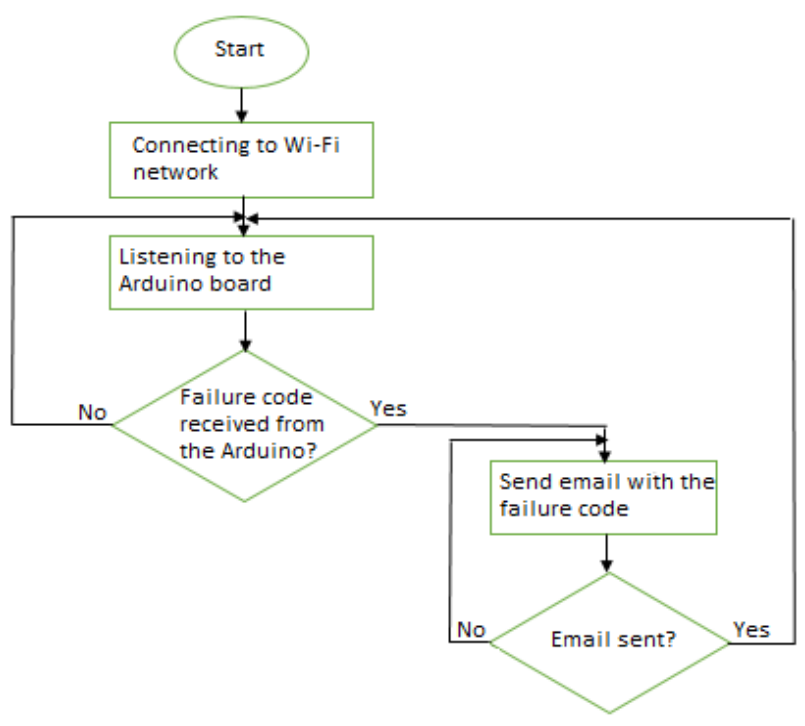

Figure 7. Flowchart of NodeMCU

\section{RESULTS AND DISCUSSION}

To test our monitoring device, we have developed a simulator of the diagnostic interface port of the helium compressor. This simulator allows us to test the performance of our monitoring system and check if there is an error in its operation before installing it in an MRI cooling system. The diagnostic interface simulator represented in Figure 8 is a network of switches linked to pins of a female DB25 connector; each switch is responsible for simulating an error corresponding to the pin connected to it.

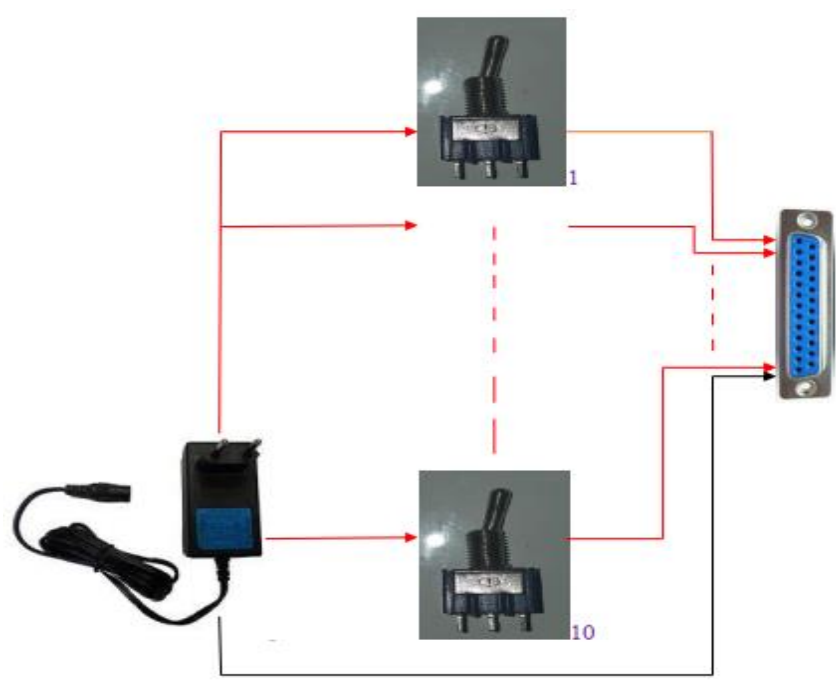

Figure 8. Diagram of the simulator

\subsection{The quench scenario}

In the case of the quench, the MRI machine will lose all the helium as an evaporated gas so fast which will create a sudden decrease in temperature and a significant increase in humidity in the surrounding air of the MRI machine. Our system can detect those massive changes in air parameters due to the DHT22 
sensor, then send an alarm SMS to the maintenance team as shown in Figure 9 and an email that includes the site name as represented in Figure 10. To test this scenario, we use a helium sprayer or azote sprayer to spatter the DHT22 sensor.

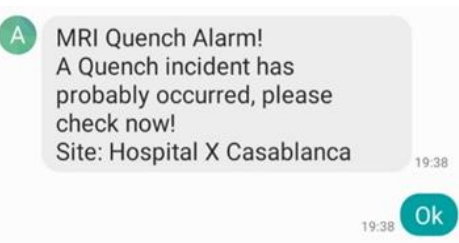

Figure 9. Quench notification SMS

\author{
MRI Quench Alarm Inbox \\ Test1 YaQin 19:34 \\ to me $\checkmark$ \\ MRI Quench Alarm! \\ A Quench incident has probably occurred, please \\ check now! \\ Site: Hospital X Casablanca
}

Figure 10. Quench notification email

\subsection{Scenario of a failure without the quench}

If there's no quench incident, our proposed system will continue monitoring the diagnostic interface pins to detect any state change which, means a failure has happened. To simulate this scenario, we have connected our system to the simulator of the diagnostic interface via a DB25 cable. When we turn on a switch, its pin will carry a voltage of $24 \mathrm{~V}$, which means a failure has occurred, our system will detect it and send a notification SMS with the specific failure code that occurred and the site name, as shown in Figure 11. It will also send an email to ensure the message is delivered, as demonstrated in Figure 12.

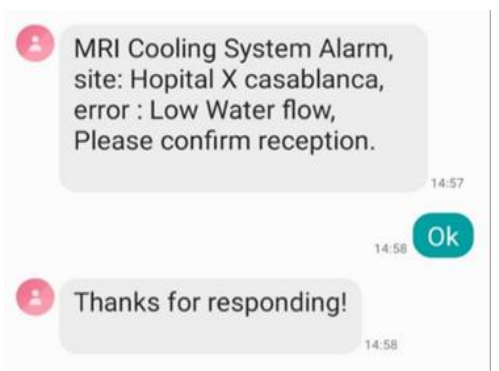

Figure 11. Failure notification SMS

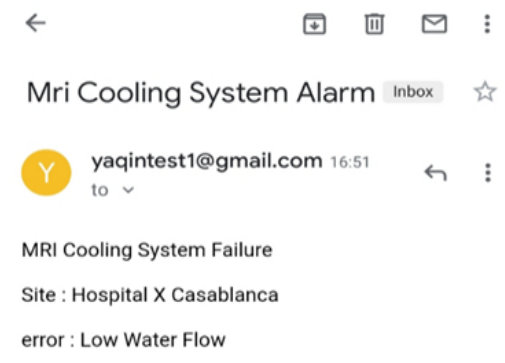

Figure 12. Failure notification email

\subsection{Disscution and future applications}

Our system has shown promoted results in the tests phase. It can be installed in just a few minutes and start monitoring the MRI cooling system in some easy steps. Despite those results, what makes it the best solution among other existing commercial systems? To find the answer, we have compared it with other systems, as represented in Table 2.

Table 2. Comparison between our system and other systems

\begin{tabular}{cccccc}
\hline System & Efficiency & Complexity & Installation & Notification & Price \\
\hline Cold Trac & High & Complex & Hard & SMS \& emails & High \\
Z-Pulse & High & Complex & Hard & SMS \& emails & High \\
Da Vinci & High & Complex & Hard & SMS \& emails & High \\
IGE system & High & Complex & Hard & SMS \& emails & High \\
Chiller fault text notification & Low & Simple & Easy & SMS & Low \\
Our system & High & Simple & Easy & SMS \& emails & Low \\
\hline
\end{tabular}

As we see in Table 2, our system is the only efficient solution with a low price in the list; it is simple with no required training to manipulate and easy to install in a few moments. Moreover, it's an internet of things (IoT) solution that sends emails as well as SMS in case of failure. We have found that we can integrate 
machine learning algorithms into our system. In case of failure, our system sends emails which we can store in the cloud. Besides, our system can detect quench incidents automatically, which will allow us to create a database of the failures that happened before unexpected Quenches and then analyze them according to a well-chosen machine learning model [22]-[24].

The objective behind the implementation of machine learning is to have the possibility to predict failures before they happen according to well-determined learning models [25]-[27]. Thus, assessing the performance of the cooling system by analyzing the history of events. Besides, artificial intelligence with deep learning models will allow us to find-if possible-a direct relationship between the behavior of the MRI cooling system and the quench incidents [28]-[31].

\section{CONCLUSION}

The MRI cooling system is the most critical part of MRI systems, as a shutdown of the cooling operation interrupts the whole process of MRI examinations. In this paper, an advanced monitoring system has been proposed to keep an eye on the cooling system and alert the technical team in case of a failure to repair the system very quickly. This proposed system will efficiently prevent the hospital from losing the expensive liquid helium and from the happening of quench incidents.

\section{REFERENCES}

[1] J. K. Mohajer, A. Nisbet, E. Velliou, M. Ajaz, and G. Schettino, "Biological Effects of Static Magnetic Field Exposure in the Context of MR-Guided Radiotherapy," The British Journal of Radiology, vol. 92, no. 1094, p. 20180484, 2019, doi: 10.1259/bjr.20180484.

[2] R. Kueng et al., "Towards MR-guided electron therapy: Measurement and simulation of clinical electron beams in magnetic fields," Physica Medica, vol. 78, pp. 83-92, 2020, doi: 10.1016/j.ejmp.2020.09.001.

[3] J. Allison and N. Yanasak, "What MRI Sequences Produce the Highest Specific Absorption Rate (SAR), and Is There Something We Should Be Doing to Reduce the SAR During Standard Examinations ?," American Journal of Roentgenology, vol. 205, no. 2, 2015, doi: 10.2214/ajr.14.14173.

[4] G. Gourzoulidis et al., "Occupational Electromagnetic Fields exposure in Magnetic Resonance Imaging systems - Preliminary results for the RF harmonic content," Physica Medica, vol. 31, pp. 757-762, 2015, doi: 10.1016/j.ejmp.2015.03.006.

[5] S. Sammet and C. Sammet, "Implementation of a comprehensive MR safety course for medical students," Journal of magnetic resonance imaging, vol. 42, pp. 1478-1486, 2015, doi: 10.1002/jmri.24993.

[6] N. Tsuchiya et al., "Safety of repeated hyperpolarized helium 3 magnetic resonance imaging in pediatric asthma patients," Pediatric Radiology, vol. 50, pp. 646-655, 2020, doi: 10.1007/s00247-019-04604-0.

[7] D. Patel et al., "Solid cryogen: a cooling system for future MgB2 MRI magnet," Scientific Reports, vol. 7, 2017, doi: 10.1038/srep43444.

[8] M. Parizh, Y. Lvovsky, and M. Sumption, "Conductors for commercial MRI magnets beyond NbTi: requirements and challenges," Superconductor Science and Technology, vol. 30, p. 014007, 2017, doi: 10.1088/0953-2048/30/1/014007.

[9] W. Huang et al., "Multifunctional luminescent magnetic cryocooler in a Gd5Mn2 pyramidal complex," Chemical Communications, vol. 54, pp. 4104-4107, 2018, doi: 10.1039/c8cc00433a

[10] T. K. F. Foo et al., "Lightweight, compact, and high-performance 3 T MR system for imaging the brain and extremities," Magnetic Resonance in Medicine, vol. 80, pp. 2232-2245, 2018, doi: 10.1002/mrm.27175.

[11] T. Baig et al., "Conceptual designs of conduction cooled MgB2 magnets for 1.5 and $3.0 \mathrm{~T}$ full body MRI systems," Superconductor Science and Technology, vol. 30, p. 043002, 2017, doi: 10.1088/1361-6668/aa609b.

[12] N. M. Cross, M. N. Hoff, and K. M. Kanal, "Avoiding MRI-Related Accidents: A Practical Approach to Implementing MR Safety," Journal of the American College of Radiology, vol. 15, pp. 1738-1744, 2018, doi: 10.1016/j.jacr.2018.06.022.

[13] D. Zhang et al., "Quench, Normal Zone Propagation Velocity, and the Development of an Active Protection Scheme for a Conduction Cooled, R\&W, MgB2 MRI Coil Segment," Superconductor Science and Technology, vol. 32, p. 125003, 2019, doi: 10.1088/1361-6668/ab48cd.

[14] C. Yao and Y. Ma, "Superconducting materials: Challenges and opportunities for large-scale applications," iScience, vol. 24, p. 102541, 2021, doi: 10.1016/j.isci.2021.102541.

[15] A. S. Beam et al., "Safety Resources and Processes in MR Imaging Departments," Radiologic Technology, vol. 90, pp. 225-236, 2019, PMID: 30635455.

[16] L. K. Knigh and P. Chan, 2005; Method and apparatus for monitoring superconducting magnet data, 6,838,964.

[17] J. D. Orndorff and S. Hope, 2014; Cryogenic sensor readout module, 2014/0238048.

[18] J. P. Roman, S. S. Josephson, and D. M. Davenport, 2003; Wireless monitoring of a mobile magnet, 6,621,413.

[19] M. J. Dalchau, R. P. Gore, R. Heinrichs, T. B. Husband, and P. A. Charles Walton, 2009; Intelligent helium compressor, $2009 / 0082657$.

[20] T. O'Reilly and A. Webb, "Deconstructing and reconstructing MRI hardware," Journal of Magnetic Resonance, vol. 306, pp. 134-138, 2019, doi: 10.1016/j.jmr.2019.07.014.

[21] Sumitomo: "F-70H and F-70L Helium Compressors Operating Manual," 2008.

[22] G. A. Susto, A. Schirru, S. Pampuri, S. McLoone, and A. Beghi, "Machine Learning for Predictive Maintenance: A Multiple Classifier Approach," IEEE Transactions on Industrial Informatics, vol. 11, pp. 812-820, 2015, doi: 10.1109/tii.2014.2349359.

[23] P. Moens et al., "Scalable Fleet Monitoring and Visualization for Smart Machine Maintenance and Industrial IoT Applications," Sensors, vol. 20, p. 4308, 2020, doi: 10.3390/s20154308.

[24] M. Nkomo, G. P. Hancke, A. M. Abu-Mahfouz, S. Sinha, and A. J. Onumanyi, “Overlay Virtualized Wireless Sensor Networks for Application in Industrial Internet of Things: A Review," Sensors, vol. 18, p. 3215, 2018, doi: 10.3390/s18103215.

[25] A. A. Shamayleh, M. Awad, and J. Farhat, "IoT Based Predictive Maintenance Management of Medical Equipment," Journal of Medical Systems, vol. 44, 2020, doi: 10.1007/s10916-020-1534-8 
[26] R. B. Patil, M. A. Patil, V. Ravi, and S. Naik, "Predictive modeling for corrective maintenance of imaging devices from machine logs," 39th Annual International Conference of the IEEE Engineering in Medicine and Biology Society (EMBC), 2017, doi: 10.1109/embc.2017.8037163.

[27] T. P. Carvalho, F. A. A. M. N. Soares, R. Vita, R. da P. Francisco, J. P. Basto, and S. G. S. Alcalá, "A systematic literature review of machine learning methods applied to predictive maintenance," Computers \& Industrial Engineering, vol. 137, p. 106024, 2019, doi: 10.1016/j.cie.2019.106024.

[28] K. Liulys, "Machine Learning Application in Predictive Maintenance," Open Conference of Electrical, Electronic and Information Sciences (eStream), 2019, doi: 10.1109/estream.2019.8732146.

[29] N. Amruthnath and T. Gupta, "A research study on unsupervised machine learning algorithms for early fault detection in predictive maintenance," 2018 5th International Conference on Industrial Engineering and Applications (ICIEA), 2018, doi: 10.1109/iea.2018.8387124.

[30] M. Hamlich and M. Ramdani, "Data classification by SAC Scout Ants for Clustering algorithm," Journal of Theoretical and Applied Information Technology, vol. 55, pp. 66-73, 2013.

[31] T. Ishida, I. Nitta, D. Fukuda, and Y. Kanazawa, "Deep Learning-Based Wafer-Map Failure Pattern Recognition Framework," 20th International Symposium on Quality Electronic Design (ISQED), pp. 291-297, 2019, doi: 10.1109/isqed.2019.8697407.

\section{BIOGRAPHIES OF AUTHORS}

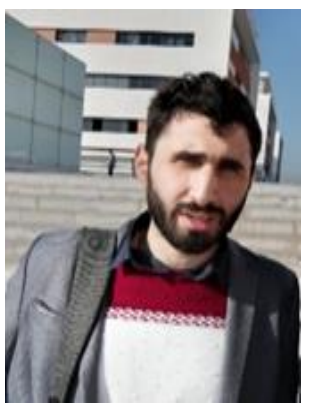

Oussama Elallam (D) SI SC P is a Biomedical R\&D engineer who graduated from University Mohammed six of health sciences in 2019, He is pursuing a PhD degree in Artificial Intelligence for medical diagnosis and care at The National Superior School of Arts and Crafts, Casablanca. Oussama has developed many biomedical devices in telemedicine and point of care testing. His areas of research are IoT, artificial intelligence in healthcare, medical wearable devices, and embedded devices. He can be contacted at email: elallamoussama7@gmail.com

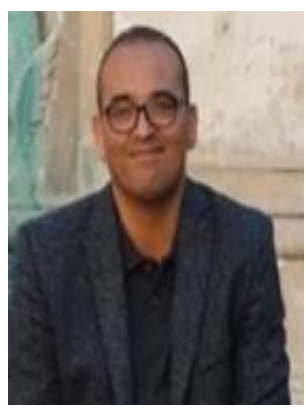

Dr. Mohamed Hamlich (D) SC P is Professor-Researcher at ENSAM (UH2C) Casablanca, Morocco since 2014. Currently, he is director of the research laboratory: "Complex Cyber Physical Systems". He is the author of several research papers in the field of machine learning, robotics and electrical energy. He can be contacted at email: moha.hamlich@gmail.com 